\section{Chitosan biopolymer: Alternative adhesion factor and scaffold matrix for 2D and 3D neuronal cultures}

\author{
Donatella Di Lisa, ${ }^{1}$ \\ Mariateresa Tedesco, ${ }^{1}$ Elena Dellacasa, ${ }^{1}$ \\ Mattia Pesce, ${ }^{2}$ Tiziano Catelani, ${ }^{3}$ \\ Paolo Massobrio, ${ }^{1}$ Roberto Raiteri, ${ }^{1}$ \\ Sergio Martinoia, ${ }^{1}$ Laura Pastorino ${ }^{1}$ \\ 1Department of Informatics, \\ Bioengineering, Robotics and System \\ Engineering, University of Genoa, \\ Genoa; ${ }^{2}$ Nanoscopy and Nikon Centre, \\ Istituto Italiano di Tecnologia, Genoa; \\ ${ }^{3}$ Electron Microscopy Facility, Istituto \\ Italiano di Tecnologia, Genoa, Italy
}

\begin{abstract}
The increase of different types of cell cultures, which can be used for the in vitro studies of physiological and/or pathological processes, has introduced the need to improve culture techniques through the use of materials and culture media that promote growth, recreating a cellular micro-environment that can be asserted in in vivo condition. The standard methods for the functionalization of supports used for cell cultures are based on the use of synthetic or natural biopolymers, which generally have high costs, such as poly-lysine and polyornithine. The aim of this work is to demonstrate the alternative use of the polysaccharide chitosan as adhesion factor and structural component for 2D/3D neuronal cultures. Thanks to its versatility, it could be easily functionalized for the fabrication of personalized of in vitro models.
\end{abstract}

\section{Introduction}

Cell cultures are fundamental for a wide of applications involving both research and industries. The increase of different types of cell cultures, which can be used for the in vitro studies of physiological and/or pathological processes, has introduced the need to improve culture techniques through the use of materials and culture media that promote growth, recreating a cellular microenvironment that can be asserted in in vivo condition. Therefore, it is important to design and develop new biologically sustainable methods, such as to contribute to the "closer-to-in vivo" condition. ${ }^{1}$

Related to that, in this work, we present the biopolymer Chitosan (CHI) as support for $2 \mathrm{D}$ and $3 \mathrm{D}$ neuronal cell cultures. Chitosan is a copolymer of glucosamine and $\mathrm{N}$-acetyl-glucosamine, obtained by the deacetylation of chitin; it is well known for its low-cost, biocompatibility, biodegradability, muco-adhesiveness, antibacterial activity as well as its bioaffinity. ${ }^{2}$

\section{Materials and Methods}

CHI was dissolved in $0.1 \mathrm{M}$ acetic acid at different concentrations $(0.01 \%-2 \%$ w/v); $2 \%$ sodium hydroxide solution. For 2D cultures only, Poly- ornithine (PORN) solution $0.15 \mathrm{mg} / \mathrm{mL}$ in water, as control.

2D: Chitosan nanometric films were obtained by dip coating.

3D: Chitosan microspheres were fabricated by a phase-inversion process using an aerodynamic encapsulator.

Chitosan films and microspheres were then used as support for the in vitro growth of primary neuronal cells. To validate the ability of chitosan to support neuronal adhesion, networks development and the differentiation capacity, morphological and functional characterization were carried out by confocal, transmission electronic and atomic force microscopies. A preliminary electrophysiological characterization of spontaneous activity was conducted by MicroElectrode Arrays (Figure 1).
A

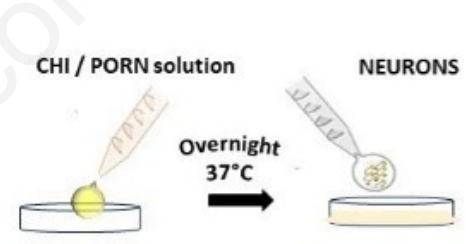

D

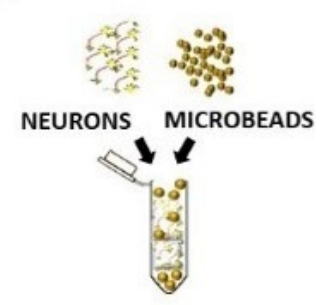

Correspondence: Laura Pastorino, Department of Informatics, Bioengineering, Robotics and System Engineering, University of Genoa, Genoa, Italy.

E-mail: Laura.pastorino@unige.it

Key words: Chitosan; adhesion factor; scaffold; neuronal network.

Contributions: DDL and MT contributed equally to this work.

Conference presentation: this paper was presented at the Second Centro 3R Annual Meeting - 3Rs in Italian Universities, 2019 , June 20-21, University of Genoa, Italy.

Received for publication: 28 October 2019 Accepted for publication: 11 November 2019.

This work is licensed under a Creative Commons Attribution NonCommercial 4.0 License (CC BY-NC 4.0).

CCopyright: the Author(s), 2019

Licensee PAGEPress, Italy

Biomedical Science and Engineering 2019; 3(s3):107 doi:10.4081/bse.2019.107

\section{Results}

Chitosan films showed the ability to support the adhesion and differentiation of neuronal culture. The growth of neurons plated on chitosan films is comparable with
Figure 1. A) Scheme of adhesion factor deposition for 2D cultures. B-C) Hippocampal culture development on dip-coating chitosan and on poly-ornithine staining staining for MAP 2 (green) and TAU (red) at 7 days in vitro; D) Scheme of 3D cell cultures assembly. E) Confocal microscope images of $3 D$ neural network at DIV 25 on $2 \%$ CHI microbeads labeled for MAP-2 (green), Tubulin BIII (red) and DAPI (blu). F) Low-mag TEM micrograph of a portion of chitosan scaffold with the neuronal network: neuritic processes inside microbeads.

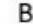

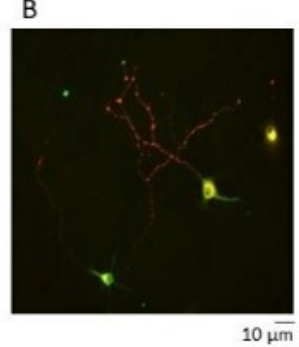

E

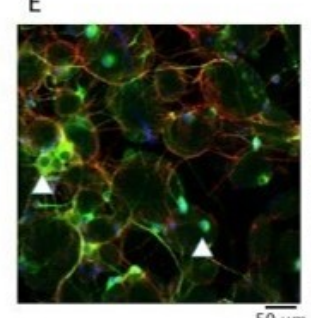

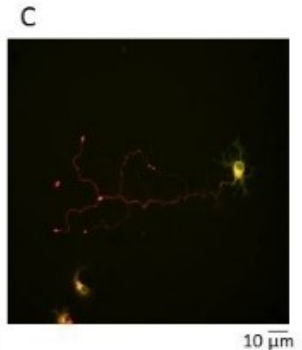

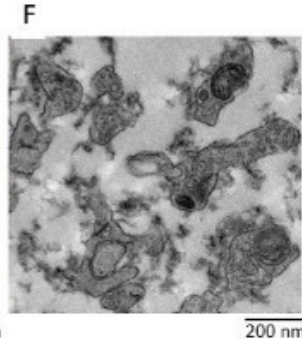


ones on standard adhesion factors (polyornithine). Furthermore, it is noted that 3D cultured neurons, show distinct morphologies that are more representative of the in vivo environment. In particular, these results have been confirmed by a preliminary electrophysiological characterization. ${ }^{3}$

\section{Conclusions}

We successfully demonstrate the alternative use of the polysaccharide chitosan as adhesion factor and structural component for $2 \mathrm{D} / 3 \mathrm{D}$ neuronal cultures. Thanks to its low cost and versatility, it could be easily functionalized for the fabrication of personalized of in vitro models.

\section{References}

1. Letourneau PC. Possible roles for cellto-substratum adhesion in neuronal morphogenesis. Dev Biol 1975;44:7791.
2. Scanga VI, Goraltchouk A, Nussaiba N, et al. Biomaterials for neural-tissue engineering - Chitosan supports the survival, migration, and differentiation of adult-derived neural stem and progenitor cells. Can J Chem 2010;88:27787.

3. Tedesco MT, Di Lisa D, Massobrio P, et al. Soft chitosan microbeads scaffold for 3D functional neuronal networks. Biomaterials 2018;156:159-71. 